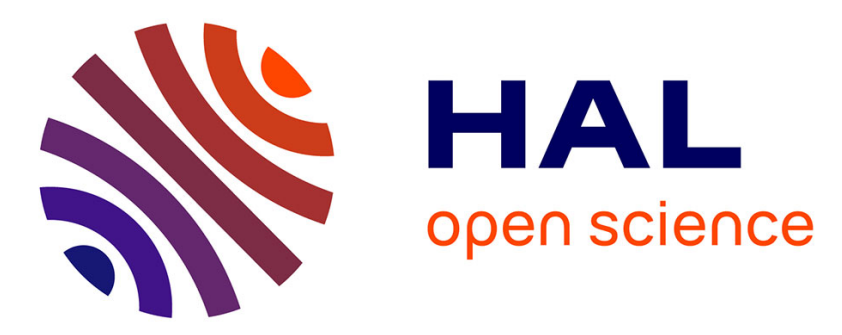

\title{
Etude des modes propres et de la réponse forcée d'une éolienne offshore flottante soumise à une houle de direction croisée par rapport à celle du vent
}

\author{
Maxime Philippe, Aurélien Babarit, Pierre Ferrant
}

\section{- To cite this version:}

Maxime Philippe, Aurélien Babarit, Pierre Ferrant. Etude des modes propres et de la réponse forcée d'une éolienne offshore flottante soumise à une houle de direction croisée par rapport à celle du vent. 12es Journées de l'Hydrodynamique, Nov 2010, Nantes, France. hal-02144477

\author{
HAL Id: hal-02144477 \\ https://hal.science/hal-02144477
}

Submitted on 30 May 2019

HAL is a multi-disciplinary open access archive for the deposit and dissemination of scientific research documents, whether they are published or not. The documents may come from teaching and research institutions in France or abroad, or from public or private research centers.
L'archive ouverte pluridisciplinaire HAL, est destinée au dépôt et à la diffusion de documents scientifiques de niveau recherche, publiés ou non, émanant des établissements d'enseignement et de recherche français ou étrangers, des laboratoires publics ou privés. 
$12^{\text {èmes }}$ Journées de l'Hydrodynamique

Nantes, $17-19$ novembre 2010

\author{
ÉTUDE DES MODES PROPRES ET DE LA RÉPONSE \\ FORCÉE D'UNE ÉOLIENNE OFFSHORE FLOTTANTE \\ SOUMISE À UNE HOULE DE DIRECTION CROISÉE PAR \\ RAPPORT A CELLE DU VENT.
}

\title{
NATURAL MODES AND FORCED MOTIONS OF AN OFFSHORE WIND TURBINE WITH DIRECTIONAL WIND AND WAVES
}

\author{
M. PHILIPPE*, A. BABARIT*, P. FERRANT* \\ * Laboratoire de Mécanique des Fluides \\ École Centrale de Nantes, BP 92101, 44321 NANTES Cedex 3 \\ maxime.philippe@ec-nantes.fr
}

\section{Résumé}

Cette étude présente une analyse dynamique d'un système composé d'une éolienne, d'un flotteur, et d'un système d'ancrage, afin d'étudier l'influence sur le système, d'une direction de houle croisée par rapport à celle du vent. Les efforts hydrodynamiques sont calculés par la théorie linéaire en domaine fréquentiel. Les efforts aérodynamiques sont pris en compte en augmentant l'amortissement et la raideur hydrodynamique par un amortissement aérodynamique et une raideur gyroscopique. Une analyse modale du système permet de connaître les fréquences propres et les modes propres du système amorti. Ceci permet d'expliquer les mouvements couplés obtenus en régime forcé; en particulier cela permet de comprendre quels modes sont excités par quelles fréquences et quelles directions de houle.

\section{Summary}

A coupled dynamic analysis of a floating wind turbine system has been performed to investigate effect of wave direction relative to wind on the system. Hydrodynamic loads are calculated by linear frequency domain approach and aerodynamic effect is taken into account by increasing hydrodynamic damping and restoring matrices with aerodynamic damping and gyroscopic stiffness. A modal analysis of the system was made to explain the calculated motions. It brings out the natural frequencies, natural modes and modal damping coefficients of the system. Excitation of natural modes, by waves explains the motion observed in the response amplitude operators, and the effect of wave direction relative to wind. This modal analysis helps to better understand the behaviour of floating wind turbine systems. 


\section{$\underline{\text { I - Introduction }}$}

Dans le cadre du développement de l'éolien offshore, l'utilisation de supports flottants et ancrés, au lieu de fondations plantées dans le sol, permet d'envisager la construction de parcs éoliens, en eau profonde. Pour ces éoliennes flottantes, les mouvements d'oscillations de la nacelle doivent être minimisés afin de limiter la fatigue des différents composants. Or, on s'attend à ce que les effets de couplage entre les mouvements induits par les vagues et les mouvements induits par la turbine soient importants et nécessitent d'être pris en compte de manière fine dans le dimensionnement des machines.

Des études ont déjà été réalisées pour simuler le comportement de ces systèmes éoliens flottants. Parmi celles-ci, certaines modélisent les efforts hydrodynamiques sur la plateforme flottante par une approche linéaire en domaine fréquentiel. Les efforts transmis par l'éolienne sur le flotteur sont modélisés par un amortissement aérodynamique et une raideur gyroscopique. [1]-[2].

Cette étude utilise la même méthode que [1]-[2], avec une approche plus rigoureuse de la prise en compte de l'influence de l'éolienne sur le flotteur. Les simulations réalisées permettent d'étudier l'effet de la directionnalité de la houle par rapport à celle du vent sur les mouvements d'un système éolien flottant. Une analyse modale du système complète le calcul des fonctions de transfert en mouvement (RAOs). Elle permet d'avoir accès aux modes propres du système et aux couplages entre ses différents degrés de liberté.

\section{II - Description du cas étudié}

Le système est supposé être animé d'un mouvement de solide rigide. Ces mouvements sont décrits dans le repère représenté sur la figure 1. L'origine du repère est placée dans le plan de la surface libre au repos et est alignée verticalement avec le centre de gravité de la plateforme. Cette origine est la référence pour les mouvements du système qui sont décrits par les degrés de liberté : cavalement (1), embardée (2), pilonnement (3), roulis (4), tangage (5), lacet (6).

La direction du vent est choisie alignée avec l'axe $x$, la direction de la houle fait un angle $\beta$ avec celle du vent. La direction $\beta=0$ représente une houle venant des $x$ négatifs, c'est à dire alignée avec la direction du vent.

Les simulations sont réalisées avec une vitesse de vent de $11.2 \mathrm{~m} . \mathrm{s}^{-1}$, qui est la vitesse de vent pour laquelle la poussée du vent exercée sur la turbine étudiée est maximale.

Le modèle d'éolienne utilisé pour cette étude est connu sous le nom de « NREL offshore 5MW baseline wind turbine $\gg[3]$. Ce concept a été développé au National Renewable Energy Laboratory afin de servir de concept de référence pour le développement de l'éolien offshore. Il ne correspond pas à une éolienne existante, c'est une représentation réaliste d'une éolienne à trois pâles de 5 MW. Les principales propriétés de cette éolienne sont données au tableau 1; ce concept

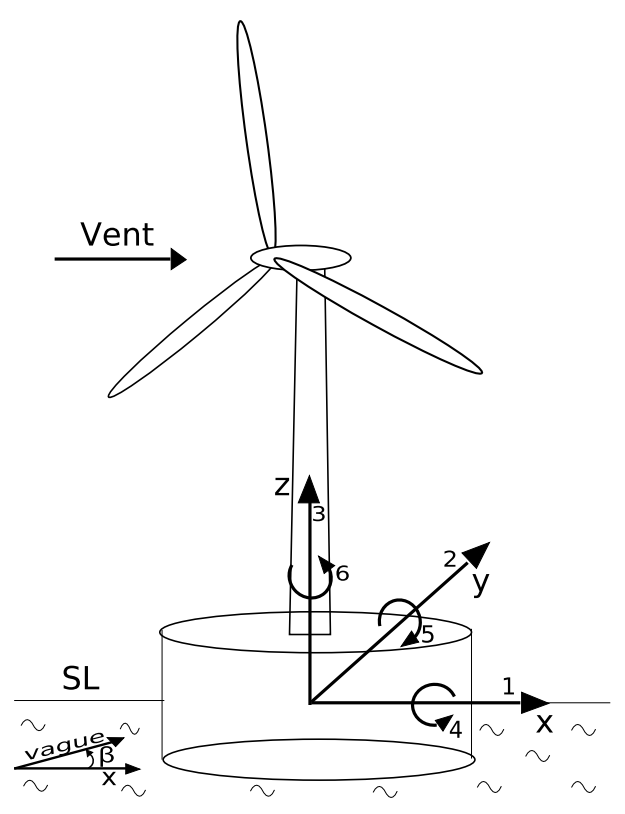

Figure 1 - Repère utilisé est décrit en détails dans [3]. 


\begin{tabular}{|l|r|}
\hline Puissance nominale & $5 \mathrm{MW}$ \\
\hline Configuration du rotor & 3 pales ; face au vent \\
\hline Diamètre du rotor & $126 \mathrm{~m}$ \\
\hline Hauteur du moyeu & $90 \mathrm{~m}$ \\
\hline Masse du rotor & $110000 \mathrm{~kg}$ \\
\hline Masse de la nacelle & $240000 \mathrm{~kg}$ \\
\hline Masse de la tour & $347460 \mathrm{~kg}$ \\
\hline Coordonnées du CdG & $(-0.2 \mathrm{~m}, 0.0 \mathrm{~m}, 64.0 \mathrm{~m})$ \\
\hline
\end{tabular}

TABLE 1 - Propriétés de l'éolienne étudiée

L'éolienne est montée sur le concept de plateforme flottante connu sous le nom de «MIT/NREL Shallow Drafted Barge ». Ce concept a été développé par E. N. Wayman, sous la direction de P. D. Sclavounos, pour supporter l'éolienne « NREL offshore 5-MW baseline wind turbine $\gg$. La plateforme $\ll$ MIT/NREL SDB $\gg$ est une barge cylindrique. Cette géométrie se base sur le moment d'inertie de la surface de flottaison pour apporter de la stabilité au système. La barge est placée dans une profondeur d'eau de $200 \mathrm{~m}$.

La géométrie de cette barge a été pensée de manière à offrir une stabilité suffisante sans ancrage. En conséquence les ancrages utilisés ne servent qu'à éviter la dérive du système. L'action des ancrages sur le système est modélisée par une matrice de raideur. Les seuls termes non nuls de cette matrice de raideur d'ancrage sont les raideurs en cavalement et en embardée. Ils valent $4 \cdot 10^{6} \mathrm{~N} . \mathrm{m}^{-1}$. Le système d'ancrage n'apporte pas de raideur en lacet au système. Toutefois sa raideur en lacet n'est pas nulle grâce aux effets aérodynamiques. Le tableau 2 liste les caractéristiques de la plateforme utilisée pour cette étude.

\begin{tabular}{|l|r|}
\hline Diamètre & $36 \mathrm{~m}$ \\
\hline Tirant d'eau, tirant d'air & $5 \mathrm{~m}, 4.5 \mathrm{~m}$ \\
\hline Masse, hauteur du ballast & $4153 \cdot 10^{3} \mathrm{~kg}, 1.595 \mathrm{~m}$ \\
\hline Masse totale & $4519 \cdot 10^{3} \mathrm{~kg}$ \\
\hline Cote du CdG & $-3.9 \mathrm{~m}$ \\
\hline Inerties en roulis et tangage /t au CdG & $3.9 \cdot 10^{8} \mathrm{~kg} \cdot \mathrm{m}^{-2}$ \\
\hline Inertie en lacet /t au CdG & $7.5 \cdot 10^{8} \mathrm{~kg} \cdot \mathrm{m}^{-2}$ \\
\hline
\end{tabular}

TABLE 2 - Caractéristiques principales de la plateforme étudiée

La géométrie de cette plateforme, à savoir une surface de flottaison importante, convient bien avec le type de modélisation des efforts hydrodynamiques choisi pour cette étude. En effet, le domaine de validité de l'approche linéaire en domaine fréquentiel impose que les dimensions du flotteur soit grandes devant l'amplitude de la houle.

\section{$\underline{\text { III - Description de la méthodologie }}$}

\section{$\underline{\text { III - } 1 \text { Approche globale de l'étude }}$}

Cette étude est composée de deux parties, une analyse dynamique et une analyse modale. L'analyse dynamique consiste à calculer les fonctions de transferts en mouvements 
(RAOs) du système éolien flottant sous l'action de la houle incidente. Cette analyse dynamique reprend la méthodologie développée par [2], en y apportant certaines améliorations, en particulier en ce qui concerne l'obtention des propriétés linéarisées représentatives de l'éolienne. Ces différences sont décrites à la partie III - 3. L'analyse modale complète l'analyse dynamique et donne accès aux fréquences propres, aux modes propres et aux coefficients d'amortissements modaux de l'éolienne flottante. Cette analyse modale met en évidence les couplages entre les degrés de liberté du système et permet de mieux comprendre les mouvements de l'éolienne flottante. Les liens entre les différentes parties de cette étude sont représentés sur la figure 2

Le système, composé de l'éolienne, du flotteur et des ancrages est soumis à l'action de la houle et du vent; il est considéré comme un solide rigide. Les efforts s'appliquant sur la plateforme sont : la réaction du fluide $F_{\text {hydro }}$, les efforts de rappel des ancrages $F_{a}$, et les efforts transmis par l'éolienne $F_{\text {aero }}$. En notant $M_{p}$ la matrice d'inertie de la plateforme, et $q$ le vecteur de ses degrés de liberté, l'équation des mouvements du système est alors :

$$
M_{p} \ddot{q}=F_{\text {aero }}+F_{\text {ancrage }}+F_{\text {hydro }}
$$

La première étape de l'étude consiste à calculer la position d'équilibre en régime permanent du système. C'est la position autour de laquelle il va osciller.

Les efforts hydrodynamiques sur la plateforme sont calculés avec le code de diffraction/radiation Aquaplus développé au Laboratoire de Mécanique des Fluides [4]. Pour chaque fréquence de vaque $\omega$, Aquaplus calcule la force de radiation en terme de masse d'eau

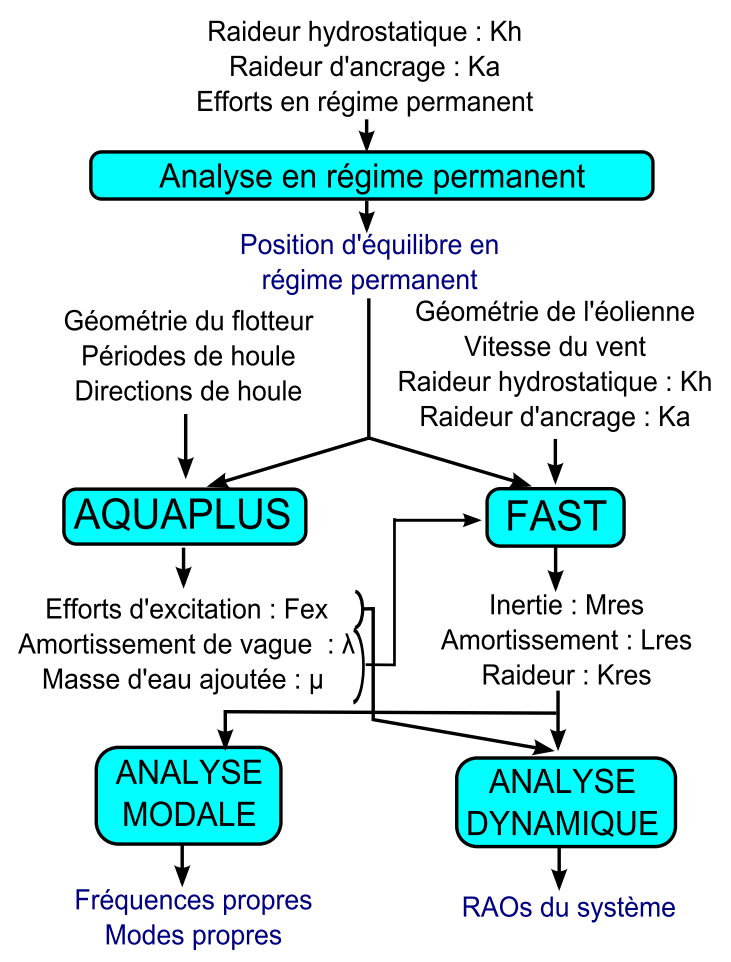

Figure 2 - Liens entre les étapes de l'étude ajoutée $\mu(\omega)$ et d'amortissement de vague $\lambda(\omega)$, ainsi que les efforts de houle incidente $F_{e x}(\omega, \beta)$. Le calcul des efforts hydrodynamiques est effectué en considérant le flotteur dans sa position d'équilibre en régime permanent, contrairement à la méthodologie suivie par [1]-[2], qui considère le flotteur en position neutre. Ceci entraine une rupture de la symétrie de la plateforme, ce qui introduit des coefficients de couplage dans les matrices des efforts hydrodynamiques. Les efforts d'ancrages sont modélisés par une matrice de raideur $K_{a}$. Les efforts hydrostatiques sont pris en compte par une matrice de raideur hydrostatique $K_{h}$.

Le code de simulation d'éolienne FAST, développé au National Renewable Energy Laboratory, permet d'obtenir une représentation linéarisée d'un modèle d'éolienne [6]. Le modèle développé avec FAST prend en compte les efforts d'ancrage, les efforts hydrostatiques et les efforts hydrodynamiques; il est linéarisé autour de la position d'équilibre en régime permanent calculé précédemment. Le modèle utilisé par FAST pour cette linéarisation est décrit en détails dans la section III - 3.

FAST donne accès aux matrices d'inertie $M_{\text {res }}$, d'amortissement $L_{r e s}$, et de raideur $K_{\text {res }}$ représentatives du système complet linéarisé autour de son point de fonctionnement en régime permanent. Finalement l'équation régissant les mouvements du système, sous 
l'action d'une houle incidente, autour de sa position d'équilibre en régime permanent, s'écrit en utilisant la notation complexe :

$$
\left(-\omega^{2} M_{r e s}(\omega)+i \omega L_{r e s}(\omega)+K_{r e s}(\omega)\right) \Delta q=F_{e x}
$$

Les matrices $M_{\text {res }}, L_{\text {res }}, K_{\text {res }}$ dépendent de de la fréquence $\omega$ de la houle incidente. $\Delta q$ est le vecteur de déplacement du système autour de sa position d'équilibre en régime permanent. Les différentes composantes des matrices $M_{r e s}, L_{r e s}, K_{\text {res }}$ sont détaillées dans la section III - 3. La résolution de l'équation 2 pour une houle incidente de $1 \mathrm{~m}$ donne accès aux RAOs du système sous l'action de la houle.

La seconde partie de cette étude est une analyse modale du système éolien flottant. Elle consiste à résoudre l'équation 2 sans le terme de forçage afin d'obtenir les fréquences et modes propres du système. Ce calcul est détaillé dans la section III - 4

\section{$\underline{\text { III }-2 \text { Étude en régime permanent }}$}

La position d'équilibre en régime permanent est régie par l'équation :

$$
F_{\text {aeros }}+F_{a}+P+F_{\text {hydrostatique }}=0
$$

avec $F_{a}$ les efforts d'ancrages, $P$ le poids du système, $F_{\text {hydrostatique }}$ les efforts hydrostatiques s'exerçant sur la plateforme, et $F_{\text {aeros }}$ les efforts aérodynamiques en régime permanent. Ces efforts aérodynamiques en régime permanent prennent en compte la poussée sur le rotor $F_{\text {poussee }}$, et le couple exercé $C$. Ces efforts sont fournis dans [3], et peuvent être recalculés avec le code FAST. Les efforts d'ancrage étant modélisés avec une matrice de raideur d'ancrage $K_{a}$, l'équation 3 devient :

$$
\left(K_{h}+K_{a}\right) X_{S}=F_{\text {aero }}
$$

avec $X_{S}$, la position d'équilibre en régime permanent. Les efforts $F_{\text {aeros }}$ s'exprimant par : $F_{\text {aeros }}=\left(F_{\text {thrust }}, 0,0, C_{\text {torque }}, F_{\text {thrust }} \cdot z_{\text {hub }}, 0\right)^{t}$. Le modèle ne comprend aucune raideur et aucune excitation en lacet. La position d'équilibre en régime permanent en lacet est considérée nulle.

\section{III - 3 Linéarisation du système avec le code FAST}

La procédure d'obtention d'une représentation linéaire d'un modèle non linéaire d'éolienne utilisée par le code FAST est expliquée en détails dans [6]. FAST offre à l'utilisateur la possibilité de définir son propre modèle d'efforts s'exerçant sur la plateforme. Dans cette section le processus de linéarisation est détaillé pour le cas du modèle de plateforme que nous avons défini.

L'équation du mouvement résolue par FAST s'écrit :

$$
M_{F}(q, u, t) \ddot{q}+f_{F}\left(q, \dot{q}, u, u_{d}, t\right)=0
$$

avec $M_{F}$ la matrice de masse, $f_{F}$ le vecteur de forçage non linéaire, $q$ le vecteur des degrés de libertés, $u$ le vecteur des entrées de contrôle, $u_{d}$ le vecteur des entrées de perturbations de vent, et $t$ le temps.

Dans notre cas, on ne considère aucune entrée de contrôle et aucune entrée de perturbation de vent. L'équation des mouvements de l'éolienne flottante, ancrée, sans houle incidente s'écrit alors : 


$$
\left[M_{e}(q, t)+M_{p}(q, t)\right] \ddot{q}+f(q, \dot{q}, t)+F_{r a d}(q, \dot{q}, t)+F_{b}(q, t)+F_{a}(q, t)=0
$$

avec $M_{e}$ la matrice de masse de l'éolienne et $M_{p}$ la matrice de masse de la plateforme. $f$ est le vecteur de forçage dû aux efforts extérieurs exceptés :

- $F_{a}$, contribution des efforts d'ancrage,

- $F_{b}$, contribution de la flottabilité,

- $F_{r a d}$, contribution des efforts de radiation calculés avec Aquaplus.

FAST linéarise ce modèle autour de la position d'équilibre en régime permanent par perturbation :

$$
q=q_{o p}+\Delta q ; \dot{q}=\dot{q}_{o p}+\Delta \dot{q} ; \ddot{q}=\ddot{q}_{o p}+\Delta \ddot{q}
$$

Le suffixe op représente une valeur prise à la position d'équilibre en régime permanent.

L'équation des mouvements d'oscillation du système autour de sa position d'équilibre en régime permanent est obtenue en linéarisant l'équation 6 :

$$
\begin{aligned}
\underbrace{(\underbrace{\left.M_{e}\right|_{o p}+\left.M_{p}\right|_{o p}}_{(1)}+\underbrace{\left.\frac{\partial F_{r a d}}{\partial \ddot{q}}\right|_{o p}}_{(2)})}_{M_{\text {res }}} \Delta \ddot{q}(t) & +\underbrace{(\underbrace{\left.\frac{\partial f}{\partial \dot{q}}\right|_{o p}}_{(3)}+\underbrace{\left.\frac{\partial F_{\text {rad }}}{\partial \dot{q}}\right|_{o p}}_{(4)})}_{L_{\text {res }}} \Delta \dot{q}(t) \\
+ & \underbrace{(\underbrace{\left.\frac{\partial f}{\partial q}\right|_{o p}+\left.\frac{\partial M_{e}+M_{p}}{\partial q}\right|_{o p}}_{K_{\text {res }}} \ddot{q}+\underbrace{\left.\frac{\partial F_{b}}{\partial q}\right|_{o p}}_{(6)}+\underbrace{\left.\left.\frac{\partial F_{a}}{\partial q}\right|_{o p}\right)}_{(7)} \Delta q(t)=0}_{(5)}
\end{aligned}
$$

Dans l'équation 8, le terme (1) est la matrice de masse du système considéré au point de fonctionnement en régime permanent. Le terme (2) est la matrice de masse d'eau ajoutée. Le terme (3) est l'amortissement de vague, et le terme (4) l'amortissement aérodynamique. Le terme (5) est la raideur ajoutée incluant la raideur gyroscopique. Le terme (6) est la raideur hydrostatique et le terme (7) est la raideur d'ancrage. FAST calcule les matrices résultantes $M_{\text {res }}, L_{\text {res }}$ et $K_{\text {res }}$.

L'équation des mouvements d'oscillation du système autour de la position d'équilibre, dus à une excitation harmonique de houle incidente est donc :

$$
M_{r e s} \Delta \ddot{q}(t)+L_{r e s} \Delta \dot{q}(t)+K_{r e s} \Delta q(t)=F_{e x}(t)
$$

En utilisant la notation complexe $\Delta q(t)=\Delta q e^{i \omega t}$, l'équation 9 devient :

$$
\left(-\omega^{2} M_{r e s}(\omega)+i \omega L_{r e s}(\omega)+K_{r e s}(\omega)\right) \Delta q=F_{e x}
$$

avec $F_{\text {ex }}$ la force d'excitation due à une houle incidente de pulsation $\omega$. Dans l'équation 10, la dépendance en $\omega$ de la matrice $M_{r e s}$ vient de la masse d'eau ajoutée, celle de la matrice $L_{r e s}$ vient de l'amortissement de vague, et celle de la matrice $K_{\text {res }}$ vient de la dépendance en accélération de cette matrice.

Dans leur étude, Wayman et al. [1]-[2] ne prennent pas en compte les ancrages, l'hydrostatique et la radiation lors de l'étape de linéarisation des propriétés du système avec le code FAST. En conséquence ils obtiennent en sortie de FAST seulement la contribution de l'éolienne sur le système. Ils ajoutent cette contribution de l'éolienne à l'équation du mouvement de la plateforme pour obtenir les mouvements du système éolien flottant. Dans cette étude nous avons suivi une approche plus rigoureuse. En effet le terme 
d'accélération du terme (5)de l'équation 8 est calculé en prenant en compte l'ensemble des efforts s'exerçant sur le système. En conséquence, Wayman et al obtiennent des matrices représentatives de l'éolienne qui ne dépendent pas de $\omega$ contrairement à cette étude.

\section{III - 4 Analyse modale}

Les oscillations libres du système autour de sa position d'équilibre en régime permanent sont régies par l'équation :

$$
M_{r e s} \Delta \ddot{q}(t)+L_{r e s} \Delta \dot{q}(t)+K_{r e s} \Delta q(t)=0
$$

Dans l'équation 11, les matrices $L_{r e s}$ et $K_{\text {res }}$ ne sont pas symétriques à cause de la rotation du rotor qui induit un amortissement aérodynamique et une raideur gyroscopique non symétriques. L'analyse modale a été réalisée en suivant la méthodologie présentée dans [7] pour l'analyse des système tournants amortis.

Les matrices $M_{\text {res }}, L_{\text {res }}$ et $K_{\text {res }}$ dépendent de $\omega$. Afin d'obtenir des résultats précis, la résolution de l'équation 11 a été effectuée pour chaque fréquence propre en prenant les valeurs des matrices $M_{r e s}, L_{r e s}$ et $K_{r e s}$ à cette fréquence. Les fréquences propres sont calculées par itération. La résolution de l'équation 11 à la fréquence $\omega_{i}$ donne le mode $i$.

Pour chaque mode, la solution de l'équation 11 est de la forme classique 12 :

$$
\Delta q(t)=\gamma p e^{\lambda t}
$$

avec $\gamma, \lambda$, et $p$ des valeurs complexes décrivant respectivement une amplitude de référence, un coefficient scalaire, et un vecteur de constante. Il faut donc résoudre le problème aux valeurs propres quadratiques 13 , où $\lambda$ et $p$ sont les valeurs propres et les vecteurs propres.

$$
\left(\lambda^{2} M+\lambda L+K\right) p=0
$$

Le changement de variable suivant permet de transformer le problème aux valeurs propres précédent de taille 6x6, en un problème linéaire de taille 12x12 [7].

$$
\begin{aligned}
g & =\{q, \dot{q}\}^{T} \\
A & =\left(\begin{array}{cc}
0 & K \\
K & L
\end{array}\right) \\
B & =\left(\begin{array}{cc}
K & 0 \\
0 & -M
\end{array}\right)
\end{aligned}
$$

Le système à résoudre devient :

$$
B \dot{g}-A g=0
$$

Le problème aux valeurs propres associé est 18, et ses vecteurs propres sont de la forme $u=\{p, \lambda p\}^{T}$. $\lambda$ et $p$ sont respectivement les valeurs propres et les vecteurs propres du problème initial 13 .

$$
(A-\lambda B) u=0
$$

Les valeurs propres apparaissent en $\mathrm{n}$ pairs de valeurs complexes conjuguées $\lambda_{i}=$ $-\alpha_{i}+j \omega_{i}$ et $\bar{\lambda}_{i}=-\alpha_{i}-j \omega_{i}$. $\alpha_{i}$ et $\omega_{i}$ sont respectivement les coefficients d'amortissement modaux et les fréquences propres du système. Les vecteurs propres apparaissent en $n$ 
pairs de vecteurs conjugués $u_{i}$ et $\bar{u}_{i}$ de taille $2 \mathrm{n}$, dont les $\mathrm{n}$ premières composantes sont les vecteurs modaux $p_{i}$ et $\bar{p}_{i}$ de la structure.

On note $\rho_{i}$ le vecteur des modules de $p_{i}$ et $\bar{p}_{i}$, et $\phi_{i}$ le vecteur des arguments de $p_{i}$. Les solutions recherchées s'expriment alors sous la forme :

$$
\begin{aligned}
& q_{i j}(t)=\gamma \rho_{i j} e^{-\alpha_{i} t} e^{i\left(\omega_{i} t+\phi_{i j}\right)} \\
& \overline{q_{i j}}(t)=\gamma \rho_{i j} e^{-\alpha_{i} t} e^{i\left(\omega_{i} t-\phi_{i j}\right)}
\end{aligned}
$$

Les vecteurs $\rho_{i}$ sont les amplitudes des modes propres aux fréquences propres $\omega_{i}$, avec un coefficient d'amortissement modal $\alpha_{i}$. Les vecteurs $\Phi_{i}$ représentent les phases entre les mouvements du mode $i$.

\section{$\underline{\text { IV - Analyse des résultats }}$}

\section{$\underline{\text { IV }-1 \quad \text { Position en régime permanent }}$}

La position en régime permanent de la structure est donnée dans le tableau 3. L'angle de tangage en régime permanent est inférieur à $10^{\circ}$, angle limite au delà duquel la perte de puissance de l'éolienne est supposée devenir significative.

\begin{tabular}{|l|r|}
\hline$K_{h}(3,3)$ & $1.0 \cdot 10^{7} \mathrm{Nm}^{-1}$ \\
\hline$K_{h}(4,4), K_{h}(5,5)$ & $4.4 \cdot 10^{8} \mathrm{Nm}$ \\
\hline Steady state pitch & $9.6^{\circ}$ \\
\hline Steady state roll & $0.5^{\circ}$ \\
\hline
\end{tabular}

TABLE 3 - Position du système en régime permanent

\section{$\underline{\text { IV }-2}$ Fréquences et modes propres du système}

Les fréquences propres obtenues pour le système sont rangées dans le vecteur $\Omega$. On appelle mode 1 à 6 , les modes propres correspondant aux fréquences propres 1 à 6 , dans l'ordre dans lequel elles sont rangées dans le vecteur $\Omega$. Les colonnes de la matrice $\rho$ correspondent aux modes propres 1 à 6 , et le vecteur $\alpha$ représente les coefficients d'amortissement modaux associés à ces modes. La matrice $\Phi$ représente les phases associées aux modes propres.

$$
\begin{gathered}
\Omega=\left(\begin{array}{c}
0.9 \\
0.7 \\
0.8 \\
0.4 \\
0.3 \\
0.4
\end{array}\right) ; \rho=\left(\begin{array}{cccccc}
0.4 & 0.8 & 0.0 & 0.0 & 0.7 & 0.0 \\
0.0 & 0.0 & 0.8 & 0.7 & 0.1 & 0.7 \\
0.6 & 0.2 & 0.0 & 0.0 & 0.2 & 0.0 \\
0.0 & 0.0 & 0.4 & 22.8 & 3.3 & 21.8 \\
0.3 & 0.5 & 0.0 & 1.5 & 32.0 & 1.4 \\
0.0 & 0.0 & 0.1 & 28.9 & 0.7 & 29.3
\end{array}\right) \\
\alpha=\left(\begin{array}{c}
0.1 \\
0.0 \\
0.0 \\
-0.2 \\
0.0 \\
0.3
\end{array}\right) ; \Phi=\left(\begin{array}{ccccccc}
178.2 & 0.0 & -3.7 & -17.3 & 180.0 & -15.3 \\
-7.5 & -167.0 & 0.0 & 0.0 & 56.6 & 0.0 \\
0.0 & -18.1 & -117.7 & -40.3 & -173.5 & -144.3 \\
-11.9 & -168.6 & -11.8 & 158.1 & -125.2 & -161.5 \\
-10.4 & 167.2 & 164.4 & 13.5 & -179.5 & 64.6 \\
-117.8 & 76.1 & -13.2 & -125.3 & -164.2 & 129.0
\end{array}\right)
\end{gathered}
$$

Les six fréquences propres du système se trouvent dans l'ensemble des fréquences qui peuvent être excitées par la houle. Le premier mode propre à 0.9 rad.s ${ }^{-1}$ correspond essentiellement à un mouvement de pilonnement qui est couplé avec un mouvement de tangage et un mouvement de cavalement. Le second mode propre à $0.7 \mathrm{rad} . \mathrm{s}^{-1}$ est similaire au premier, avec les mêmes couplages, mais est dominé par le cavalement. Ces deux modes ont un couplage entre les mouvements de cavalement, pilonnement et tangage. Ce couplage 


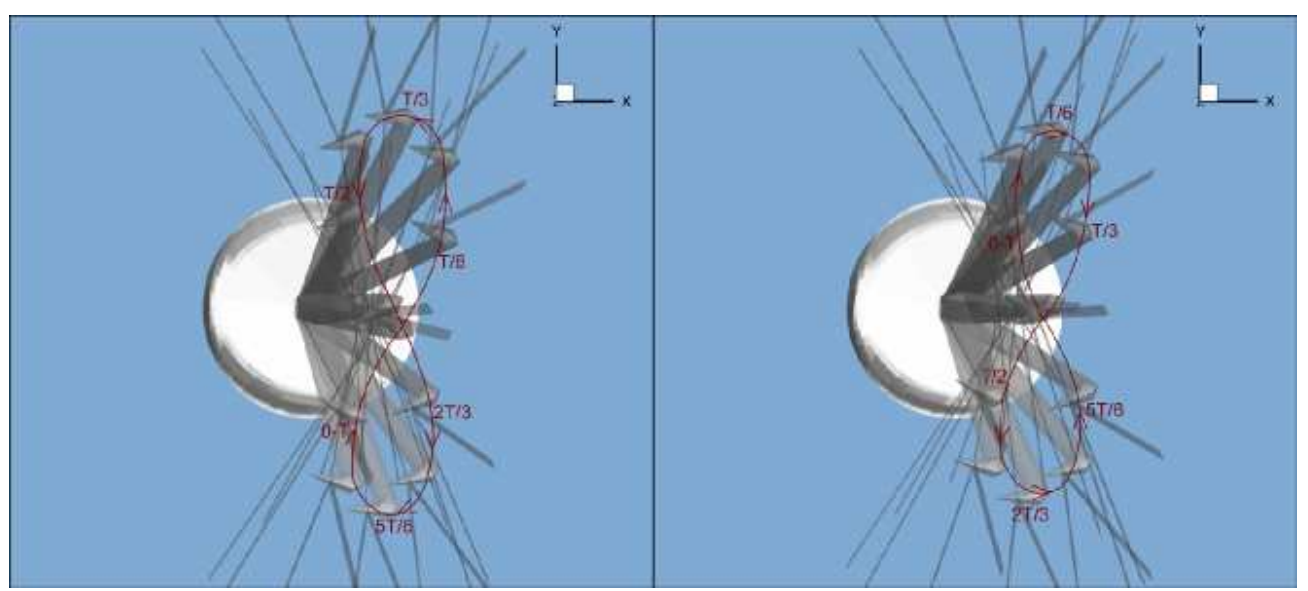

Figure 3 - Visualisation des modes propres 4 (à gauche) et 6 (à droite)

entre les degrés de liberté horizontaux était attendu. Le troisième mode à $0.8 \mathrm{rad} . \mathrm{s}^{-1}$ est dominé par le mouvement d'embardée avec un couplage important avec le mouvement de roulis et un plus faible avec celui de lacet. Ce couplage entre les degrés de liberté en rotation était lui aussi attendu. Les fréquences propres 1,2 et 3 sont proches, les modes propres correspondants peuvent être excités en même temps. Le cinquième mode est dominé par le tangage qui est faiblement couplé avec les cinq autres degrés de liberté. Le couplage avec les mouvements de translation cavalement, embardée, pilonnement est négligeable. En revanche le couplage tangage-roulis est observable avec une valeur de $10 \%$. Ce couplage n'était pas attendu.

Les valeurs des fréquences propres 4 et 6 sont les mêmes, $0.4 \mathrm{rad} . \mathrm{s}^{-1}$. Les amplitudes des modes propres correspondants sont approximativement les mêmes, mais les phases entre les mouvements sont différentes. Le coefficient d'amortissement modal du mode 4 est négatif, ce qui est le signe d'une possible instabilité [7]. Les modes 4 et 6 sont représentés sur la figure 3. Ces modes montrent un couplage important entre les mouvements de roulis et de lacet. Les phases entre les mouvements n'étant pas les mêmes, le système oscille en sens inverse dans les deux modes, et donc ceux-ci ne peuvent être excités en même temps.

\section{$\underline{\text { IV }-3 \text { Fonctions de transferts en fonction de la direction de la houle }}$}

Les fonctions de transfert (RAOs) du système éolien flottant sont représentées sur la figure 4. Ces RAOs sont calculées pour 5 directions de houle de $0^{\circ}$ à $180^{\circ}$. La direction $0^{\circ}$ correspond à une houle provenant de la direction des x négatifs. Ces RAOs représentent la réponse du système en fonction de la période et de la direction de la houle incidente pour une vitesse de vent donnée.

La réponse en cavalement est représentée sur la figure 4a. Elle présente un large pic autour de 0.7 rad. $\mathrm{s}^{-1}$ pour toutes les directions de houle. Lorsque les vagues sont alignées avec la direction de cavalement, l'excitation du mode propre 2, dominé par le cavalement, explique l'importante réponse obtenue autour de 0.7 rad.s $\mathrm{s}^{-1}$, qui est la fréquence propre associée à ce mode. Pour les vagues venant avec un angle de $90^{\circ}$, le mouvement de cavalement obtenu n'est pas nul. En effet, à cause de la position d'équilibre en régime permanent du flotteur, celui-ci n'est pas symétrique, et la force d'excitation en cavalement pour une houle venant de $90^{\circ}$ n'est pas nulle. De plus, pour une houle venant de $90^{\circ}$ à $0.7 \mathrm{rad} . \mathrm{s}^{-1}$, la composante en pilonnement des modes 1 et 2 peut être excitée, et le système obtient alors un mouvement de cavalement du au couplage cavalement-pilonnement de ces modes.

La figure $4 \mathrm{~b}$ représente la réponse en embardée de la structure. Elle présente un pic 


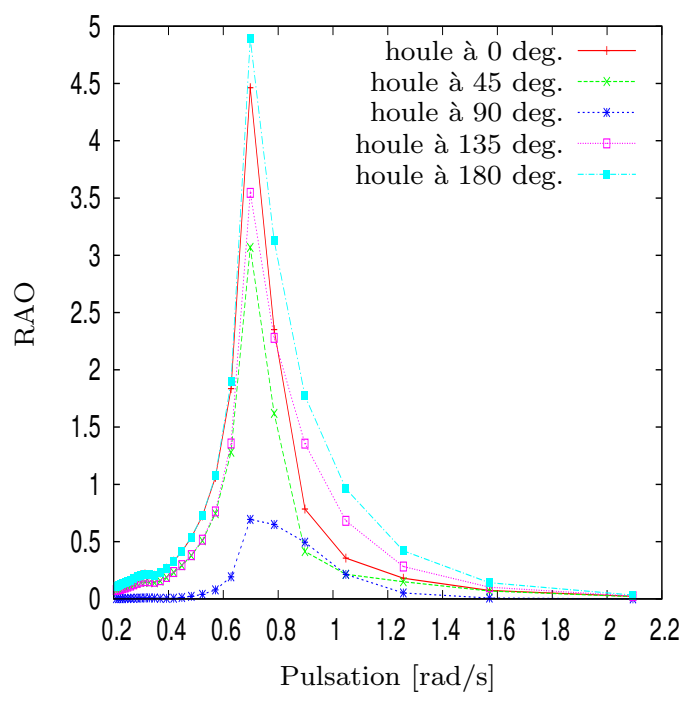

(a) cavalement

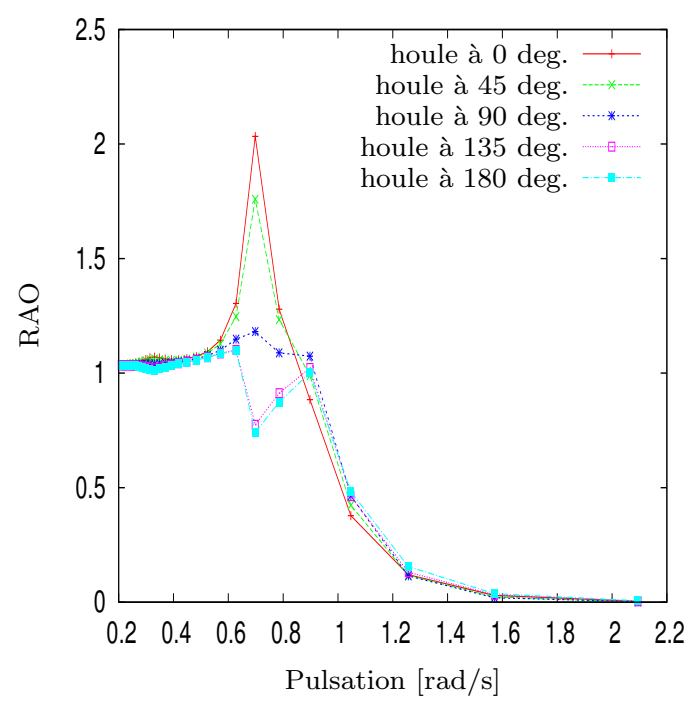

(c) pilonnement

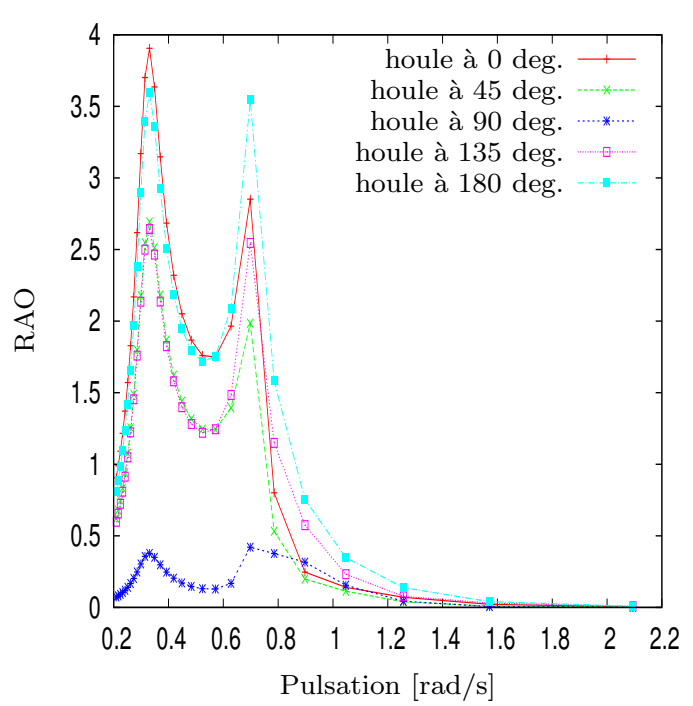

(e) tangage

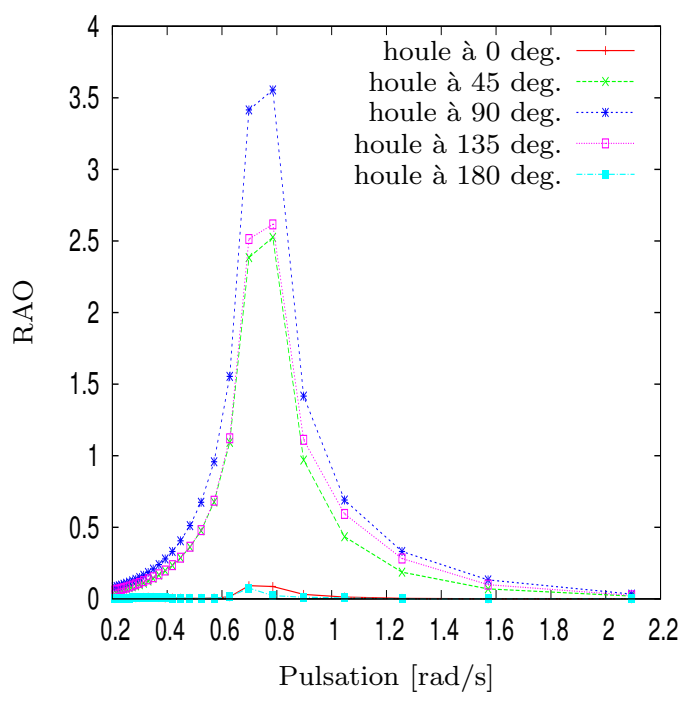

(b) embardée

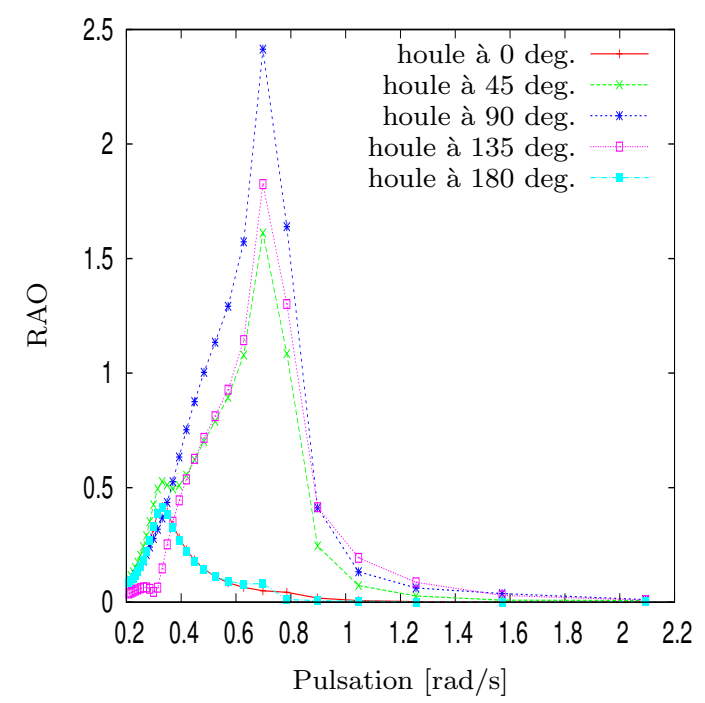

(d) roulis

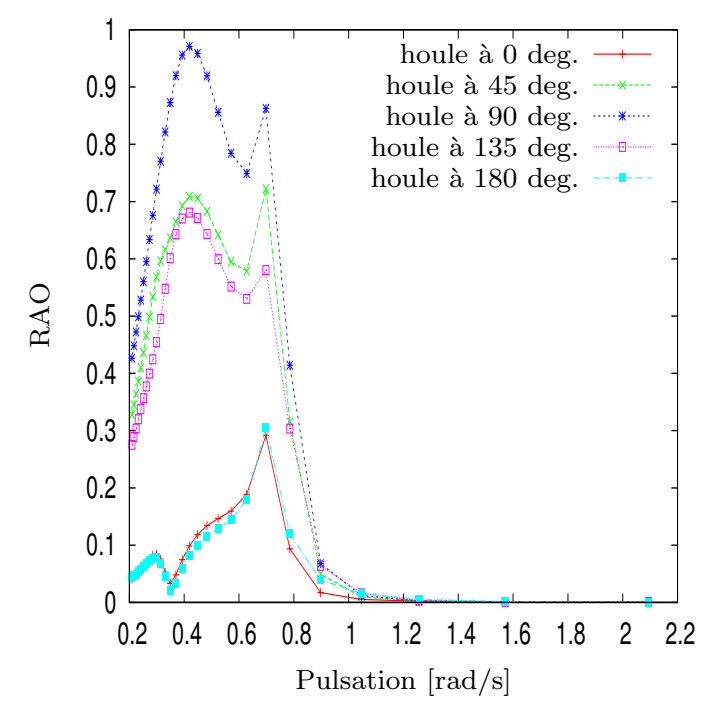

(f) lacet

Figure 4 - RAOs du système pour différentes directions de houle 
important autour de $0.8 \mathrm{rad} . \mathrm{s}^{-1}$, en revanche pour les directions $0^{\circ}$ et $180^{\circ}$, la réponse est négligeable en embardée. L'importante réponse à $0.8 \mathrm{rad} . \mathrm{s}^{-1}$ lorsque la houle est alignée avec la direction d'embardée correspond à l'excitation du mode propre 3 ayant une forte composante d'embardée. Contrairement à la réponse en cavalement, il n'y a pas de couplage pilonnement-embardée pour amener un mouvement d'embardée avec une houle venant de $0^{\circ}$ ou $180^{\circ}$; de plus la force d'excitation dans la direction d'embardée est faible pour ces directions de houle.

La figure 4c représente la RAO en pilonnement du système. Pour une houle à $0^{\circ}$, la réponse en pilonnement du système est maximale à $0.7 \mathrm{rad} . \mathrm{s}^{-1}$. Pour une houle à $180^{\circ}$, la réponse passe par un minimum local à $0.7 \mathrm{rad} . \mathrm{s}^{-1}$. La différence observée ne vient pas de la force d'excitation en pilonnement qui est globalement la même quelle que soit la direction de houle. Pour une houle à $0.7 \mathrm{rad} . \mathrm{s}^{-1}$, les modes propres 1 et 2 peuvent être excités et amenés un mouvement de pilonnement. Le mode 2 , dominé par le cavalement sera plus excité avec une houle de $0^{\circ}$ ou $180^{\circ}$. L'excitation en phase, pour la houle de $0^{\circ}$, et en opposition de phase, pour la houle de $180^{\circ}$, des modes 1 et 2 peut expliquer les maximum et minimum de réponse observés dans ces deux cas.

La réponse en roulis du système est représentée sur la figure 4d. Elle présente un large pic autour de $0.7 \mathrm{rad} . \mathrm{s}^{-1}$ pour les directions de houle $45^{\circ}, 90^{\circ}$ et $180^{\circ}$. Ce pic s'explique par l'excitation du mode propre 3 embardée-roulis-lacet. On remarque que les modes 4 et 6 n'apportent que peu de réponse autour de leur fréquence de $0.4 \mathrm{rad} . \mathrm{s}^{-1}$. Ils devraient apporter un mouvement de roulis important, mais comme ils ne sont pas en phase il semble qu'ils s'annulent et n'apportent que peu de mouvement non marqué par un pic de résonance. Pour les directions $0^{\circ}$ et $180^{\circ}$, on observe un petit pic à $0.3 \mathrm{rad} . \mathrm{s}^{-1}$. Il correspond à l'excitation du couplage tangage-roulis du mode 5 .

La RAO en tangage est représentée sur la figure 4e. Les mouvements sont représentés autour de la position en régime permanent qui est de $9.6^{\circ}$ de tangage. Les courbes de réponses présentent deux pics de réponse. Le premier autour de $0.3 \mathrm{rad} . \mathrm{s}^{-1}$ correspond à l'excitation du mode 5. Le second correspond à l'excitation des modes 1 et 2 couplés en embardée-pilonnement-tangage.

La dernière figure $4 \mathrm{f}$ représente la $\mathrm{RAO}$ en lacet du système. L'amplitude de la réponse en lacet est modérée pour toutes les directions et toutes les fréquences de houle. Il n'y a pas d'excitation en lacet du système, le mouvement en lacet vient donc du couplage avec les autres degrés de liberté de l'éolienne flottante. Ce couplage existe dans les modes propres 3, 4, 5 et 6 . Le fort couplage entre le roulis et le lacet dans les modes 4 et 6 , peut expliquer que la réponse en lacet soit plus importante lorsque la direction de la houle croise celle du vent.

\section{$\underline{\mathrm{V}-\text { Conclusion }}$}

Cette étude met en évidence l'importance de l'analyse modale pour la compréhension des mouvements des éoliennes flottantes. Les fréquences propres et modes propres qui ont été calculés permettent de mettre en évidence les couplages existants entre les degrés de liberté de l'éolienne considérée. L'effet de la direction de la houle incidente sur l'éolienne flottante a été étudié, et il est apparu que les modes propres de la structure sont excités différemment suivant la direction de la houle. Le cas où la houle et le vent ne sont pas alignés est le cas pour lequel la réponse en lacet du système est la plus importante.

Dans cette étude, l'éolienne est modélisée par un amortissement et une raideur équivalente. Il existe des outils de simulation en domaine temporel qui donnent accès à une modélisation plus complète de l'éolienne avec plusieurs degrés de liberté [6]. Il serait intéressant d'étudier 
l'effet de la direction de la houle incidente en domaine temporel, et de regarder ses effets sur les phénomènes transitoires.

\section{$\underline{\text { VI - Remerciements }}$}

Les auteurs remercient l'Agence de l'Environnement et de la Maîtrise de l'Énergie et la région Pays de la Loire pour avoir permis la mise en place de la thèse au cours de laquelle cette étude a été menée.

\section{Références}

[1] E.N. Wayman. (2006) : Coupled Dynamics and Economic Analysis of Floating Wind turbines Systems. Master Thesis, Massachusetts Institute of Technology.

[2] E.N. Wayman, P.D. Sclavounos, S. Butterfield, J. Jonkman, W. Musial. (2006) : Coupled Dynamic Modelling of Floating Wind Turbine Systems. Offshore Technology Conference, 1-4 May 2006, Houston, TX .

[3] J. Jonkman, S. Butterfield, W. Musial, G. Scott. (2009) : Definition of a 5-MW Reference Wind Turbine for Offshore System Developpement. NREL/TP-500-39060, Golden, CO : NREL.

[4] G. Delhommeau. (1993) : Seakeeking codes AQUADYN and AQUAPLUS. In 19th WEGEMT SCHOOL Numerical Simulation of Hydrodynamics : Ships and Offshores Structures.

[5] B. Molin. (2002) : Hydrodynamique des Structures Offshore. Editions TECHNIP.

[6] J.M. Jonkman, M.L. Buhl. (2005) : FAST User Guide. Golden, CO : National Renewable Energy Laboratory.

[7] T. Gmür. (1997) : Dynamique des structures : analyse modale numérique. Lausanne : Presses polytechniques et universitaires romandes.

[8] J. Jonkman. (2007) : Dynamics Modelling and Load Analysis of an Offshore Floating Wind Turbine. Ph.D. Thesis, Department of Aerospace Engineering Sciences, University of Colorado, Boulder, CO;NREL/TP-500-41958, Golden, CO :NREL. 